\title{
CORRECTION TO POINCARE COMPLEX THICKENINGS AND CONCORDANCE OBSTRUCTIONS
}

\author{
BY J. P. E. HODGSON
}

It has been pointed out to me that a calculation of D. Sullivan contradicts Theorem 3.3 of my paper Poincare complex thickenings and concordance obstructions [1]. The calculation indicates the presence of 2-torsion in the homeotopy group of $S^{9} \times S^{11}$. In fact my proof of Theorem 3.3 depended on a mistake in the computations with the $J$-homomorphism. It is quite easy to see that there is an element of order 2 in the kernel of $\pi_{9}(\mathrm{PL}) \rightarrow \pi_{9}(\mathrm{~g})$ which would give rise to an element of order 2 in $J T^{22}\left(S^{9} \vee S^{11}\right)$, for in this case $\pi_{\theta}(\mathrm{PL})$ is of order 16 , from the exact sequence $0 \rightarrow \pi_{\theta}(0) \rightarrow \pi_{\theta}(\mathrm{PL})$ $\rightarrow \Gamma_{\theta} \rightarrow 0$ and $\pi_{\theta}(\mathcal{G})$ is of order 8 .

\section{REFERENCE}

1. J. P. E. Hodgson, Poincare complex thickenings and concordance obstructions, Bull. Amer. Math. Soc. 76 (1970), 1039-1043.

University of Pennsylvania, Philadelphia, Pennsylvania 19104

AMS 1969 subject classifications. Primary 5518, 5701, 5720.

$K e y$ words and phrases. Homeotopy group, $J$-homomorphism. 